\title{
Synthesis of new biheterocyclic 4-(2-(1,3,4-oxadiazol-2- yl)ethyl)-6-(trifluoromethyl)pyrimidines
}

\author{
Juliana L. Malavolta ${ }^{a},{ }^{\star}$, Alex F. C. Flores ${ }^{a}$, Rayane B. Goularte ${ }^{a}$, Alynne A. Souto ${ }^{a}$, \\ Morgana Doneda $^{a}$
}

Departamento de Química, Universidade Federal de Santa Maria, Santa Maria, RS, Brasil

*julimana@hotmail.com

Keywords: pyrimidines, hydrazides, oxadiazoles

\section{INTRODUCTION}

Among the heterocyclic compounds pyrimidines and oxadiazoles stand out because of their biological and medicinal importance. Pyrimidines have been used as antibiotics, antineoplastic, among others. ${ }^{1}$ On the other hand, 1,3,4-oxadiazoles have been identified as the main core of many bioactive molecules exercising antiinflamatory, antimicrobial and anticonvulsant activities. ${ }^{2}$

In recent years we have focused our interest on methyl 7,7,7-trihalo-4-methoxy-6-oxo-3-heptenoates. These represent significant and versatile halogencontaining building block for the synthesis of heterocyclic systems, which often show high biological activities. ${ }^{3}$

In connection with our studies on the synthesis of azole and pyrazine derivatives we were interested in developing general and convenient methods for the synthesis of biheterocyclic systems from methyl 7,7,7-trihalo-4-methoxy-6-oxo-3-heptenoates. Here we report the synthesis of 4-(2-(1,3,4-oxadiazol-2yl)ethyl)-6-trifluoromethylpyrimidines (6-10) from 3(6-trifluoromethylpyrimidin-4-yl)propanehydrazide and aromatic aldehydes.

\section{RESULTS AND DISCUSSION}

Methyl 7,7,7-trifluoro-4-methoxy-6-oxo-3-heptenoate 1 was synthesized by an acylation method early described the in literature. ${ }^{3}$ The cyclocondensation of 1 with benzamidine and S-methylthiourea was investigated. The reaction proceeded in methanol with $\mathrm{NaOH}$ for 1 hour at room temperature to give the respective pyrimidines $\mathbf{2}$ and $\mathbf{3}$ in good yields. ${ }^{4}$ Treatment of methyl 3-(6-trifluoromethylpyrimidin-4yl)propanoates $\mathbf{2}$ and $\mathbf{3}$ with hydrazine hydrate in refluxing methanol afforded the corresponding hydrazides $\mathbf{4}$ and $\mathbf{5}$. Hydrazides $\mathbf{4}$ and $\mathbf{5}$ were reacted with aromatic aldehydes in ethanol:water solution under catalysis by $\mathrm{NaHSO}_{3}$ to afford the target biheterocyclic title systems 6-10 (Scheme 1). Compounds 2-10 are new, and were synthesized via simple and unexpensive methods. All synthesized compounds were obtained in good yields and high purity as solids and their structures were attributed by ${ }^{1} \mathrm{H} /{ }^{13} \mathrm{C}$ NMR and $\mathrm{GC} / \mathrm{MS}$ data.

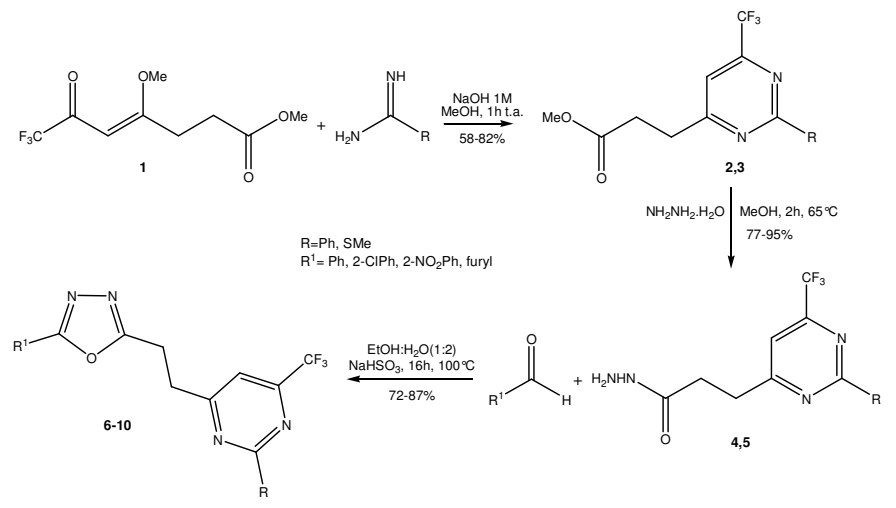

Scheme 1. Synthesis of 4-(2-(1,3,4-oxadiazol-2-yl)ethyl)6 -(trifluoromethyl)pyrimidines

\section{CONCLUSION}

In summary, this work shows a highly efficient and versatile synthetic route for the obtention of biheterocyclic systems with high potential activity. We use easily manipulable inexpensive reagents in relatively short reaction times producing new trifluoromethyl-containing derivatives in good yields.

\section{ACKNOWLEDGEMENTS}

We are grateful for the financial support from $\mathrm{CNPq}$ (Grant 476158/2007-9), CAPES and FAPERGS.

\section{REFERENCES}

${ }^{1}$ Katritzky and Rees. Comprehensive Heterocyclic Chemistry,

Vol.1-8, 1 Pergamon Press, Oxford, $1^{\text {st }}$ ed.1984, $2^{\text {nd }}$ ed. 1995.

2 Jaiprakash, N. S.; Aniruddha, R. C.; Devanand, B. S., Bioorg. Med. Chem. Lett. 2011, 21, 444.

${ }^{3}$ Flores, A. F. C.; Flores, D. C.; Oliveira, G.; Pizzuti, L.; da Silva, R. M. S.; Martins, M. A. P.; Bonacorso, H. G. J. Braz. Chem. Soc. 2008, 19, 184. Flores, A. F. C.; Pizzuti, L.; Piovesan, L. A.; Flores, D. C.; Malavolta, J. L.; Pereira, C. M. P. Tetrahedron Lett. 2010, 51, 4908.

${ }^{4}$ Flores, A. F. C.; Pizzuti, L.; Brondani, S.; Rossato, M.; Zanatta, N.; Martins, M. A. P. J. Braz. Chem. Soc. 2007, 18, 1316. 